
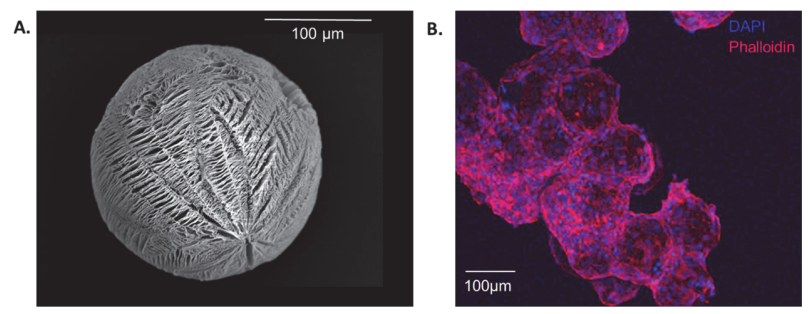

Abstract BS28 Figure 1 (A) Scanning electron microscopy image of the microcarrier. (B) iPSC attachment to the surface of TIPS microspheres, 24 hours post-seeding

0.0001) (figure 1B). The attached cells proliferated (doubling time $\leq 24$ hrs, $n=3$ ). iPSC attached to the microcarriers retained their pluripotent phenotype, demonstrated by positive expression of markers, SOX2, OCT4, TRA-1-60 and SSEA4. Pluripotency was further demonstrated by differentiation of TIPS-iPSC into a mixed cardiomyocyte-like population exhibiting a beating phenotype for up to 40 days in culture and positive expression of cardiac markers Troponin $\mathrm{T}$ and sarcomeric $\alpha$-Actinin.

Conclusion This work demonstrates that TIPS microcarriers offer a supporting matrix for culturing and differentiating iPSC and may provide an injectable biomaterial for cardiac regeneration.

Conflict of Interest N/A

\section{BS29 SINGLE CELL TRANSCRIPTOMICS REVEALS REGENERATIVE EMBRYONIC PATHWAYS LOST IN THE ADULT EPICARDIUM}

Vincent Knight-Schrijver, Laure Gambardella, Hongorzul Davaapil, Sanjay Sinha, Alexander Ross, Ludovic Vallier. University of Cambridge, Cambridge, UK

\subsection{6/heartjnl-2021-BCS.227}

Background Damage to the heart muscle often leads to chronic arrhythmia and heart failure. This is because the muscle of the adult human heart does not functionally regenerate after injury and is replaced by fibrous non-contractile and non-conductive scar tissue. However, the heart muscle of some vertebrates and developing mammals is capable of regeneration which restores the muscular function of the damaged tissue. Evidence suggests that the epicardium plays a crucial role in orchestrating this successful regenerative response in animals like the zebrafish and that epicardium cells are active in developmental stages of life. However, in adult humans the epicardium is putatively quiescent.

Objectives We hypothesise that the difference in the regenerative capacity of human adult and foetal myocardium may be explained in part by differences in the epicardium and reactivation of these molecular processes lost in adult epicardium may lead to effective myocardial regeneration. Using single-cell RNA sequencing, our aim was to capture the regenerative epicardial signalling present in the foetal epicardium that may be absent in adult hearts.

Methods Heart sections from 7 human embryos between 8 and 12 weeks of gestation were dissociated and sequenced with scRNA-seq. ScRNA-seq data from 5 human adult hearts between 55 and 75 years old were acquired from the Heart Cell Atlas. Data were integrated and epicardial cells in all samples were identified using supervised classification and canonical markers.
Results We compared the markers expressed in foetal and adult mesothelial epicardial cells and identified foetal, adult and stage-independent epicardial signatures. Among these were a number of pro-angiogenic factors specific to the foetal epicardium, including a group of WNT-signalling secreted factors, WNT2B, SFRP2 and SFRP5. Gene Ontology suggested a shift towards immune response of the epicardial cells during maturation. Additionally, an epicardial cluster with fibroblast-like markers was found only in the foetal heart (96\% foetal).

We examined organ-wide influences of epicardial signalling by mapping the potential ligand receptor interactions from the epicardial-derived secreted factors to receptors upregulated in other heart cell clusters. This revealed the prospective influence of reactivating foetal epicardial pathways for heart regeneration. Lastly, we demonstrated that epicardium derived from human embryonic stem cells exhibits some of these lost regenerative pathways, promoting their value as a model and as therapeutic agents.

Conclusions We have shown that the adult epicardium has major differences to the foetal epicardium. Firstly, a set of epicardium-specific angiogenic pathways are absent from the adult epicardium and secondly, foetal hearts contain another epicardial cell type that is not present in the adult heart. Recapitulating both the missing epicardial signals and the synergy between the foetal epicardial cell types may be essential for developing effective regenerative stem cell therapy.

Conflict of Interest None

\section{BS30 LIGHT SHEET IMAGING TO ANALYSE THE SPATIAL DISTRIBUTION OF PROTEINS IN ATHEROSCLEROTIC PLAQUES}

Ziqi Zhou, Nicholas Van-Hateren, Sheila Francis, Maria Fragiadaki, Paul Evans. The University of Sheffield, Sheffield, UK

\subsection{6/heartjnl-2021-BCS.228}

The mechanisms linking shear stress, endothelial physiology and plaque biology are currently poorly understood, but their elucidation could identify new strategies to reduce plaque growth and rupture. To address this, we use eNOS as high shear stress marker coupled to immunofluorescent staining, optical clearing and light-sheet microscopy, to develop a system for analysing the spatial distribution of proteins in murine plaques and correlate them with local WSS.

Results Confocal microscopy of concavity mounted slides revealed strong eNOS staining at the outer curvature of WT mice but significantly reduced staining at the inner curvature $(\mathrm{N}=5 ; \mathrm{P}<0.001)$. This is consistent with high WSS induction of eNOS because the outer curvature corresponds to a HSS site (Suo et al) however precise correlations between eNOS and shear stress could not be made because the tissue geometry was lost during processing.

Light-sheet microscopy of cleared samples with preserved 3D structure confirmed elevated expression of eNOS at HSS regions of the outer curvature (figure $1 \mathrm{~A} ; \mathrm{N}=5 \mathrm{WT} ; \mathrm{P}<0.01$ ). In aortic arches of ApoE-/- mice, eNOS was observed at the outer curvature but was also present at portions of atherosclerotic plaques (figure 1B). Further analysis revealed that eNOS expression was higher at the proximal (upstream) part 